\title{
Our experience: Surgical tracheostomy in 20 patients with COVID- 19 pneumonitis
}

\author{
Elinor Warner ${ }^{1}$, Julia Hadley ${ }^{1}$, Nabeel Bhatti ${ }^{1}$, Helen Drewery ${ }^{1}$, Alistair Mulcahy ${ }^{1}$, Rishi \\ Bhandari $^{1}$, and Khalid Ghufoor ${ }^{2}$ \\ ${ }^{1}$ Barts and The London NHS Trust \\ ${ }^{2}$ Royal London Hospital
}

May 11, 2020

Our experience: Surgical tracheostomy in 20 patients with COVID- 19 pneumonitis

\section{Key points}

1. The potential for transmission of the SARS -CoV-2 virus to healthcare workers performing the tracheostomy necessitates changes to standard tracheostomy procedures

2. Surgical tracheostomy performed in a theatre environment enables surgical and anaesthetic teams to support critical care staff by taking ownership of this aspect of patient care

3. All ENT and Maxillofacial teams used PPE including FFP3 facemasks, visors, and disposable hoods

4. Median (interquartile range) day to tracheostomy after intubation was 19 days (16-26.3). Median follow up was 17.5 days (range $3-24$ ).

5. None of the healthcare workers involved were clinically or PCR positive for COVID -19 in the postoperative period

Keywords SARS-CoV-2, Novel Coronavirus, COVID-19, Tracheostomy, Mechanical Ventilation

\section{Introduction}

The ongoing COVID-19 pandemic has resulted in unprecedented pressure on critical care and requirements for mechanical ventilation. Tracheostomy (both percutaneous and surgical techniques) play an established role in mechanically ventilated patients to aid weaning, decrease sedation, and facilitate step down to level two and ward- based care. In the COVID-19 setting, the situation continues to rapidly evolve, with recommendations to date based on expert opinion rather than robust evidence.

The increased demand for performing tracheostomies, either surgically or percutaneously, in COVID -19 presents problems due to the potential for aerosol generation. Here we share our experience in formulating a COVID -19 tracheostomy standard operating procedure (SOP) to mitigate that risk, and the outcomes of our first 18 surgical tracheostomies in COVID-19 patients.

\section{Initial steps}

A multidisciplinary steering group, initiated by consultants from the Adult Critical Care Unit (ACCU), maxillofacial surgery and anaesthesia, and joined by ENT, created a standard operating procedure (SOP) for all tracheostomies proceeding during the pandemic period (Figure 1). Senior nursing staff were also consulted. The group considered risks to staff and patients inherent in both percutaneous and surgical techniques and how techniques might be modified to mitigate risks. Deviations from usual institutional practice were set 
out. An early version of our local SOP was subsequently adopted by the British Laryngological Association [1] and we have subsequently developed our local SOP further, based on our evolving experience (Figure 1).

\section{Technique}

Our unit has opted to undertake all tracheostomies surgically for two reasons. The group believed that control of aerosol generation could be more effective, with minor modifications to surgical and anaesthetic techniques. Secondly, the anticipated increase in workload for critical care staff during the COVID-19 pandemic led to the group opting for a surgical approach that would share the burden of managing these patients across different teams.

Usual practice when placing a surgical tracheostomy is that there are minimal interruptions to mechanical ventilation. Aerosolisation of respiratory secretions into the area surrounding the operating field is likely during endotracheal tube repositioning, tracheal window formation and exchange of endotracheal tube for tracheostomy tube. To limit the exposure of healthcare workers involved in the procedure to unnecessary risk ventilation is stopped entirely during these steps. The endotracheal tube is clamped when the ventilator circuit is broken. Suctioning of the trachea is performed using closed in-line suctioning apparatus.

\section{Human factors}

Human factors training suggests that key to reducing error is maintaining usual practice as much as possible in times of adversity [2]. Therefore only minor modifications were made to standard surgical and anaesthetic techniques for tracheostomy in order to minimise risk of aerosol generation (Figure 1). The relative merits of undertaking the procedures in a positive pressure operating theatre versus a negative pressure side room in critical care were discussed and it was felt that the safety benefits of the theatre environment outweighed the potential risks. We simulated our tracheostomy protocol on two SARS-CoV-2 negative patients prior to our first COVID-19 case.

\section{Timing}

We opted for late tracheostomies for two reasons. International experience of the care of intubated patients with COVID-19 pneumonia describes a patient group with complex ventilatory requirements, unpredictable trajectory and high acuity. Performing a tracheostomy early in the course of the illness appeared to confer high risk to the patient with questionable benefit [3]. Given the poor outcomes for many COVID-19 patients demonstrated internationally, it appeared prudent to delay tracheostomy until patients have survived the acute period, to avoid operating on patients who are unlikely to survive and exposing the theatre teams to unnecessary risk $[5,6]$. Furthermore performing early tracheostomies in these patients exposes the operating team to risk of exposure at a time SARS-CoV-2 viral load is likely to be higher. Although there is a risk of subglottic stenosis from prolonged intubation, this is thought to be only $1-2 \%$ with the low pressure cuffs now in common use [4].

\section{Materials and Methods}

Study population

Prospective data collection including adults $>18$ years undergoing elective tracheostomy from 13th April to 1st May 2020 for weaning of ventilation.

All patients had a diagnosis of COVID -19

All procedures were performed in theatre using a surgical tracheostomy technique.

The study was registered as an audit with the clinical effectiveness team

\section{Results}

Critical care admissions 
From 4th March 2020 - 3rd May 2020 there were 185 patients admitted to ACCU with COVID-19 pneumonia. Of these 122 were intubated and 20 have had tracheostomies. Sixty- three patients were never intubated. Of the intubated patients 46 were discharged alive from ACCU (38\%), whilst 53 (43\%) died and 23 remain on ACCU.

\section{Inclusion criteria}

27 patients underwent surgical tracheostomy in the time period. Seven of these were SARS-CoV-2 negative and therefore didn't meet the inclusion criteria. 20 patients were included in the analysis

\section{Demographics}

See table 1

Table 1 Patient demographics and trends in tracheostomy

\section{Timing of tracheostomy}

The median (IQR) days to tracheostomy was 19 (16-26.3). Three tracheostomies were performed at less than 14 days $(15 \%)$. The indication in 17 of 20 cases was anticipated prolonged wean. Only 3 out of 20 patients were deemed suitable for a trial of extubation prior to procedure.

\section{Ventilatory support on the day of procedure}

On the morning of tracheostomy all patients had PEEP [?]10, and 95\% had $\mathrm{F}_{\mathrm{i}} 0_{2}$ [?]0.4 in accordance with our tracheostomy SOP.

\section{Anticoagulation and inotropes}

One third of the patients were on low dose vasopressor support on the morning of the procedure.

Thirty-five percent of patients were on therapeutic anticoagulation prior to tracheostomy. In all cases this was held for 24 hours prior to the procedure. Of the remaining patients, 2 were receiving a twice daily prophylactic low molecular weight heparin due to raised D-Dimer but in the absence of confirmed thromboembolic complications.

\section{Inflammatory markers}

The average CRP was 73 on the morning of the procedure. In the majority of patients this was trending down $(67 \%)$.

Twelve (60\%) of the 20 patients had a pyrexia on the day of, or one day prior to tracheostomy.

\section{Anatomical considerations}

Two of the patients had had prior neck surgery, (central neck scars from previous tracheostomies). Forty-five percent of patients had increased neck adiposity.

\section{Technique and teams}

All tracheostomies were performed in a positive pressure operating theatre. A consultant was the primary operator for all cases.

\section{Staff safety}

Negative swabs were not required before surgery.

All surgical teams wore double surgical gloves, fluid resistant disposable surgical gown, face visor, FFP3 masks, disposable shoe covers and fluid resistant hoods.

No patients had inadvertent endotracheal tube cuff rupture during the procedure, therefore reducing surgeon exposure to aerosolised viral particles as per previously described techniques [7]. 
There have been no reported cases of the surgical or nursing teams developing symptoms post procedure.

\section{Surgical outcomes}

Median follow up was 17.5 days (range 3-24).

There were three tracheostomy related complications, bleeding 24 hours post-operatively, a damaged pilot balloon requiring change of tracheostomy tube and surgical empysema managed conservatively. One patient died (mortality rate $6 \%$ ) of multifactorial causes 36 hours after surgical tracheostomy.

Twelve of twenty patients had been successfully weaned off mechanical ventilation (median 3 days after tracheostomy). Six patients have been discharged from critical care (median 8.5 days after tracheostomy), with 2 more fit for ward discharge. Four patients have been successfully decannulated (median day 7.5 after weaning from mechanical ventilation) and one patient has been discharged from hospital.

\section{Discussion}

Local and national guidance have helped in creating best practice models for teams to adhere to across the UK, reassuring teams of their personal safety when performing aerosol generating procedures in COVID -19 patients.

The recent emergence of this disease in the UK has meant that robust outcome data is yet to be established. Although there has been a recent series of 51 patients undergoing percutaneous tracheostomy [8], there have been no published series of solely surgical tracheostomy outcomes in COVID-19.

There was good adherence to our local and national guidance for both timing and level of ventilatory support prior to tracheostomy suggesting robust processes locally to ensure tracheostomies are being performed appropriately [1].

We believe modifications to the standard surgical tracheostomy technique represents a safe technique for patients and staff alike. Although there is no definite evidence to guide which of percutaneous or surgical techniques are less aerosol generating, no staff members involved in our series or that of Takhar et al have tested positive for COVID-19 in the post-operative period [9].

Whilst this initial study is limited in terms of numbers and follow up duration, there are many positive aspects which support the continued use of the national guidance. Complication rates were low (15\%) and comparable to the published literature [10]. This early data on surgical tracheostomy outcomes, demonstrates that it is a safe technique in this cohort of patients and should help to give hospitals around the country confidence to continue to perform this procedure, via the technique they are most comfortable with, whilst further evidence is collected.

Words 1476

\section{References}

1. Guidelines | BLA [Internet]. Britishlaryngological.org. 2020 [cited 6 May 2020]. Available from:https://www.britishlaryngological.org/resources/guidelines2. Al Yaghchi C, Ferguson C, Sandhu G. Percutaneous tracheostomy in patients with COVID-19: sealing the bronchoscope with an in-line suction sheath. British Journal of Anaesthesia. 2020 3. Young D, Harrison D, Cuthbertson B, Rowan K, TracMan Collaborators f. Effect of Early vs Late Tracheostomy Placement on Survival in Patients Receiving Mechanical Ventilation. JAMA. 2013;309(20):2121. 4. Leung C. Clinical features of deaths in the novel coronavirus epidemic in China. Reviews in Medical Virology. 2020. 5. Grasselli G, Zangrillo A, Zanella A, Antonelli M, Cabrini L, Castelli A et al. Baseline Characteristics and Outcomes of 1591 Patients Infected With SARS-CoV-2 Admitted to ICUs of the Lombardy Region, Italy. JAMA. 2020;323(16):1574. 6. Miles B, Schiff B, Ganly I, Ow T, Cohen E, Genden E et al. Tracheostomy during COV-SARS-CoV -2 pandemic: Recommendations from the New York Head and Neck Society. Head \& Neck. 2020;. 7. Thiruchelvam J, Cheng L, Drewery H. How to do a safe tracheostomy Technical note. International Journal of Oral and Maxillofacial Surgery. 2008;37(5):484-486. 8. Takhar A, Walker A, Tricklebank S, Wyncoll D, Hart N, 
Jacob $\mathrm{T}$ et al. Recommendation of a practical guideline for safe tracheostomy during the COVID-19 pandemic. European Archives of Oto-Rhino-Laryngology. 2020;. 9. Takhar A, Tornari C, Amin N, Wyncoll D, Tricklebank S, Arora A, Ahmad I, Simo R. Percutaneous tracheostomy in COVID-19 pneumonitis patients requiring prolonged mechanical ventilation: Initial experience in 51 patients and preliminary outcomes Authorea. April 27, 2020. DOI:10.22541/au.158801954.4753876210. Lipton G, Stewart M, McDermid R, Docking R, Urquhart C, Morrison M et al. Multispecialty tracheostomy experience. The Annals of The Royal College of Surgeons of England. 2020;102(5):343-347.

\section{Hosted file}

table 1 surgical tracheostomy.pptx available at https://authorea.com/users/319941/articles/ 449605-our-experience-surgical-tracheostomy-in-20-patients-with-covid-19-pneumonitis

\section{Hosted file}

figure 1.pptx available at https://authorea.com/users/319941/articles/449605-our-experiencesurgical-tracheostomy-in-20-patients-with-covid-19-pneumonitis 